\title{
COUPLING OF A BOUSSINESQ WAVE MODEL WITH A MOORED SHIP BEHAVIOR MODEL
}

\author{
Liliana Pinheiro ${ }^{1}$, Conceição Fortes ${ }^{2}$, João Santos ${ }^{3}$ and José Fernandes ${ }^{4}$
}

\begin{abstract}
A set of procedures to evaluate the time series of the diffraction forces on a moored ship inside a harbor basin is presented. Nonlinear wave propagation is obtained using a Boussinesq finite element numerical model, BOUSSWMH. Determination of hydrodynamic forces acting on the ship is achieved using a modified version of the WAMIT model. Finally, time series of the wave forces on the ship and of the inherent motions of the moored ship are obtained using BAS numerical model. The main focuses of this work are: the coupling of these three models and the modification of the method used in WAMIT to determine diffraction forces. Some simple and practical applications of this procedure are presented as well.
\end{abstract}

Keywords: Moored ship behavior, nonlinear wave propagation

\section{INTRODUCTION}

Sea waves inside a sheltered basin can cause excessive movement of a ship at its berth which can lead not only to interruption of loading and unloading operations but also to collisions with other ships or port infrastructures with significant economic losses.

Coupling numerical models for wave propagation and behavior of moored ships subjected to the wave action can identify potentially adverse sea states and help planning safe harbor activities.

A numerical tool called SWAMS has been developed. The great advantage of such a tool is the ability to provide time series of ship's movements, as well as of forces and extensions in the mooring elements once the sea-wave characteristics offshore are known. This information can be derived from buoys measurements or prediction models, making this a very useful tool, both for design of port infrastructures and for planning of port activities.

For sea-wave propagation SWAMS may use a linear model based upon the mild slope equation, DREAMS (Fortes, 1993), that is able to simulate the propagation of monochromatic waves into sheltered areas taking into account refraction, diffraction and reflection or a more complex model, BOUSS-WMH (Boussinesq Wave Model for Harbours, Pinheiro (2007)), that is capable of a more accurate description of sea states evolution into varying-depth sheltered regions by taking into account refraction, diffraction, partial reflection and nonlinear interactions as well as energy dissipation due to bottom friction and wave breaking.

To simulate moored ship behaviour, SWAMS uses the numerical package MOORNAV (Santos, 1994) which resorts to the frequency domain results of the WAMIT model (Korsemeyer et al. 1988) for the radiation and diffraction problems of a free floating body to get the hydrodynamic forces necessary to BAS model (Mynett et al. 1985). This latter model assembles and solves, in the time domain, the moored ship motion equations taking into account the incident sea waves and the geometry and constitutive relations of the mooring system elements.

WAMIT was developed at the former Department of Ocean Engineering of Massachusetts Institute of Technology to evaluate the wave-induced stresses on floating structures deployed offshore.

Within a harbor basin waves will certainly be diffracted by the harbor structures which invalidates the use of WAMIT model unless one considers several floating bodies, some of them immobile and occupying the whole of the liquid column. However, this implies the solution of a huge system of linear equations. A possible alternative is to use the relations established by Haskind (1957) involving the potential flow associated with the waves radiated by the ship and the potential of incident waves at the position where the ship is placed and which can be used to calculate the waves forces on the immobilized ship.

\footnotetext{
${ }^{1}$ Ports and Structures Division, National Laboratory for Civil Engineering, Av. do Brasil 101, Lisbon, 1700-066, Portugal

${ }^{2}$ Ports and Structures Division, National Laboratory for Civil Engineering, Av. Do Brasil 101, Lisbon, 1700-066, Portugal

${ }^{3}$ Civil Engineering Department, Instituto Superior de Engenharia de Lisboa, Rua Conselheiro Emídio Navarro 1, Lisboa, 1959-007, Portugal

${ }^{4}$ Mechanical Engineering Department, IST - Technical University of Lisbon, Av. Rovisco Pais 1, Lisbon, 1049-001, Portugal
} 
In this paper we describe: the components of SWAMS package, the application of this package to evaluate moored ship motions in a very special condition in which the model can be used directly with WAMIT; the new implemented procedures based on Haskind relations and the first test results obtained with these procedures. The communication ends with the presentation of final remarks on the work.

\section{SWAMS NUMERICAL TOOL}

SWAMS - Simulation of Wave Action on Moored Ships - is an integrated tool for numerical modeling of wave propagation and of the behavior of moored ships inside ports to help in the decision making process for planning port operations.

It consists of a graphical user interface and a set of modules for running numerical models. The user interface enables the storage and manipulation of data, executes the numerical models and enables the graphical visualization of results.

Each model corresponds to a module to which are attached the databases that bring together all the project information. With this application one may conduct studies with little or no knowledge on the operation of the underlying numerical models. So this application allows one to store and to manipulate data easily, to run the numerical models, to get the results and to perform the corresponding graphical representation of sea wave propagation as well as of ship response to incident sea waves in harbor basins.

SWAMS was developed in Microsoft Access ${ }^{\mathrm{TM}}$, which has the advantage of including the eventdriven object programming language Visual Basic for Applications (VBA). An advantage of this language is the possibility to use and handle different Microsoft Windows applications.

The SWAMS ensemble includes:

- SWAN module corresponding to the spectral model with the same name, SWAN, Booij et al. (1996), which is based on the equation of conservation of wave action and it is capable of modeling the non-linear propagation of sea waves;

- DREAMS module, corresponding to the numerical model DREAMS (Fortes, 1993), which is based on the mild-slope equation;

- BOUSS-WMH module, based on the nonlinear finite element model BOUSS-WMH, Pinheiro (2007), which solves the nonlinear Boussinesq equations derived by Nwogu (1993);

- MOORNAV module, Santos (1994) that assembles and solves the moored ship motion equations assuming the linearity of the floating body / waves system, as proposed by Cummins (1962).

SWAMS databases are MS Access ${ }^{\mathrm{TM}}$ databases, corresponding to the numerical models modules, which contain all the project information together with several folders where all the created files are stored.

The graphical representation of data and results in SWAMS is made with Golden Software Surfer ${ }^{\mathrm{TM}}$ (for SWAN module), with Tecplot ${ }^{\mathrm{TM}}$ (for DREAMS module) and with MS Excel TM (for WAMIT and BAS modules) and with Autocad (for WAMITmodule). All these graphical visualization programs are invoked by event-driven macros that automate the entire process of creating maps and graphs.

In the following subsections one describes only the wave propagation and the model for the response ships moored inside ports that are used in the case studies presented in this communication.

\section{DREAMS model}

The incident wave field at the site where the ship will be placed is provided by the numerical model DREAMS (Fortes, 1993). It is a model for calculating the propagation and deformation of regular waves in coastal areas and it can be applied to study short sea wave propagation into a port as well as marina resonance excited by incident long-period waves. It is based on the two-dimensional mild slope equation given by:

$$
\nabla \cdot\left(c c_{g} \nabla \eta\right)+k^{2} c c_{g} \eta=0
$$

where $\eta$ is the free-surface elevation, which is a function of the (x,y) coordinates, $c=\omega / k$ is the phase velocity or wave speed, $c_{g}=d \omega / d k$ is the group velocity, and $\omega$ is the wave angular frequency that relates to $k$, the wave number, through the dispersion relation $\omega^{2}=g k \tanh (k h)$. 
The mild-slope equation takes into account the combined effects of refraction, diffraction and reflection and describes the propagation of small amplitude monochromatic waves across regions of mild-slope bottom as happens in harbors, bays and other coastal areas.

The numerical method used to solve the mild-slope equation is the Finite Element Method (FEM). Since this model does not present any limitation as to incidence angle of the incoming wave in the calculation domain, the same domain may be used for a wide range of incident wave directions.

Boundary conditions implemented in the model are the radiation condition which allows the exit from the domain of disturbances propagating towards infinity, the combined radiation-generation condition that on open boundaries allows the entrance of incident waves and the exit of disturbances created in the domain and the (total or partial) reflection, condition that is valid for the solid contours in the study area (beaches, cliffs, piers, etc.).

The model calculates wave height indexes $(\mathrm{H} / \mathrm{Ho})$, the ratio between the wave height at the point in the calculation domain, $\mathrm{H}$, and the wave height at the entrance of the same domain, Ho, or amplification coefficients (in port resonance studies) as well as the directions of wave propagation. The horizontal velocity field at the free surface and the wave crests (equal-phase lines) are optional results.

\section{BOUSS-WMH model}

BOUSS-WMH (Walkley and Berzins 2002, Pinheiro 2007) solves the extended Boussinesq equations derived by Nwogu (1993), which are valid from shallow water up to intermediate water depths. It is a model suitable for the propagation and deformation of nonlinear and dispersive waves in areas of variable bathymetry since it reproduces the some of the most important phenomena in coastal regions of low to medium depth: wave diffraction, refraction, reflection, shoaling, as well as energy dispersion for other harmonics.

For the vertical integration of the velocity profile, the velocity at a selected value of the vertical coordinate is used. The model uses the SPRINT package (Berzins et al. 1 984) for the temporal integration and the Galerkin method with an unstructured finite-element mesh for spatial discretization. Finite-element meshes are generated with automatic generator GMALHA, Pinheiro et al. (2007), specifically created for sea-wave propagation models. For the generation of waves (regular or irregular) in the computational domain a source function is used following the Wei et al. (1999) procedure. This condition allows not only to generate waves, but also to absorb the waves reflected by the physical boundaries of the domain and which propagate towards the source, thus avoiding its permanence in the computational domain and the subsequent alteration of the wave characteristics there.

Total reflection or absorption are the boundary conditions available in the model. The condition of total absorption is achieved by placing a sponge layer. To simulate wave interaction with physical boundaries (such as breakwaters, jetties, cliffs, etc.) a total reflection boundary condition is imposed. This model was developed for one and two dimensions (in plan view) applications and its results are the free surface elevation and the horizontal velocity (representative) at each point of the computational domain.

\section{MOORNAV numerical package}

In LNEC, the evaluation of determining the ship motions while moored at a berth and subjected to incident sea waves is carried out using the numerical package MOORNAV (Santos 1994). This is essentially two numerical models:

- WAMIT (Korsemeyer et al. 1988) that solves, in the frequency domain, the radiation and diffraction problems associated to the interaction between incident waves and a free-floating body;

- BAS (Mynett et al. 1985) that assembles and solves, in the time domain, the motion equations of a moored ship at berth taking into account the time series of the wave forces on the ship, the impulse response functions of the ship and the constitutive relations of the mooring system elements (mooring lines and fenders);

to which an interface between the two models is added, since the WAMIT results are not exactly the quantities needed for the BAS model operation and because it is necessary to provide BAS with the time series of the forces exerted by the incident sea waves in addition to the wind and (near stationary) current forces. 


\section{Moored ship equations}

The ability to model the forces on the ship hull due to her interaction with the waves is critical to the establishment of the motion equations for a moored ship.

Assuming small amplitude of the ship movements along each of her six degrees of freedom, it is easy to define the part corresponding to the quasi-static variation of submerged hull form. This leads to the hydrostatic restoring matrix $C_{k j}$ whose coefficients are the force along mode $k$ due to a unit change, in still water, of the ship position along mode $j$.

The same assumption of small amplitude ship movements leads to the linearity of the interaction between the hull and the incident waves. Such linearity allows the decomposition of that problem into two simpler problems, Cummins (1962): The radiation problem in which one determines the forces along each degree of freedom that are needed for an arbitrary hull movement in otherwise calm water, and the diffraction problem in which one determines the force $F_{k}^{d}$ along each degree of freedom $k$ that is exerted by the incident sea waves on the motionless ship hull.

From the above results the equation of motion for the moored ship

$$
\sum_{j=1}^{6}\left[\left(M_{k j}+m_{k j}\right) \ddot{x}_{j}(t)+\int_{-\infty}^{t} K_{k j}(t-\tau) \dot{x}(\tau) d \tau+C_{k j} x_{j}(t)\right]=F_{k}^{d}(t)+F_{k}^{m}(t)+F_{k}^{f}(t)
$$

where $M_{k j}$ is the mass matrix of the ship and $F_{k}^{m}(t)$ and $F_{k}^{f}(t)$ are the instantaneous values of the forces due to mooring lines and fenders. Strictly speaking, this is a set of six equations whose solutions are the time series of the ship movements along each of her six degrees of freedom as well as of the efforts in the mooring lines and fenders.

In the equation above mass and hydrostatic restoring matrices depend only on the ship geometry and on the mass distribution therein. The forces due to mooring lines and the fenders can be determined from the constitutive relations of these elements of the mooring system and from the changes in the distance between their ends (in the case of fenders one has account for the no-length variation associated to the absence of contact between the ship and the fender).

The numerical model BAS (Mynett et al. 1985) assembles and solves, in the time domain, these equations of motion taking into account the time series of forces due to waves incident on the ship, the impulse response functions of the ship and the constitutive relations of the mooring system elements (mooring lines and fenders). It uses a $\theta$ method to discretize the time variations in those equations and it solves the resulting set of nonlinear algebraic equations using the Newton-Raphson method, the first estimate of the velocity at instant $n+1$ needed to apply that method being provided by first order Adams-Bashforth method.

\section{WAMIT model}

The impulse response function, the infinite-frequency added mass matrix and the excitation forces due to waves that arise in equation (2) depend on the hull shape and on the disturbance caused by the motionless hull in the wave propagation flow or on flow generated by the hull movement in otherwise calm water.

Assuming that any sea state that acts on the ship can be decomposed into sine waves of known period and direction, the diffraction force associated with this sea state can be obtained from the superposition of the stationary diffraction forces due to each of these sinusoidal components. That is, results from the diffraction problem in the frequency domain may be used to produce a time domain result.

Also the impulse response functions and the infinite-frequency added masses can be determined from results obtained in the frequency domain, in this case for the radiation problem:

$$
\begin{gathered}
K_{k j}(t)=\frac{2}{\pi} \int_{0}^{\infty} b_{k j}(\omega) \cos (\omega t) d \omega \\
m_{k j}=a_{k j}(\omega)+\frac{1}{\omega} \int_{0}^{\infty} K_{k j}(t) \sin (\omega t) d \omega
\end{gathered}
$$


where $b_{k j}(\omega)$ is the damping coefficient for the frequency $\omega$ and $a_{k j}(\omega)$ the added mass coefficient for the same frequency. From equation (4) it becomes clear the designation of $m_{k j}$ as the infinite-frequency added mass coefficient since as the frequency $\omega$ goes to infinity the second value in the sum in (4) tends to zero and $m_{k j}=a_{k j}(\infty)$. The added mass and damping coefficients result from the decomposition of the stationary force associated to the radiation problem corresponding to sinusoidal movement of frequency $\omega$ into a part that is in phase with the speed of the body motion (the damping coefficient) and a part in phase with the body acceleration (the added mass coefficient).

The use of frequency-domain results to generate data for a problem in the time domain is due to the greater availability of numerical models to solve, in the frequency domain, the interaction problem of a floating body with the waves.

The WAMIT (Korsemeyer et al. 1988) is one of these models. This is a model developed at the former Department of Oceani Engineering of the Massachusetts Institute of Technology, which uses a panel method to solve in the frequency domain the diffraction and radiation problems of a free floating body. This model is based on the Green's second equality to determine the intensity of the source and dipole distributions over the panels used in discretization of the hull wetted surface. With such distributions it is possible to generate the harmonic potentials of the flow associated with the radiation and diffraction problems of a free ship placed in a constant-depth zone not limited horizontally.

\section{Haskind relations}

The model WAMIT contemplates the existence of several floating bodies, some of which can be held still and occupy the whole of the liquid column. This would enable the direct use of this model in simulation of the radiation and diffraction problems of a ship within a sheltered basin where the wave incident on the ship may have been diffracted by the structures that protect the harbor. This detail of a possible crest curvature of the incident waves on the ship is especially important for the diffraction problem. In fact for the radiation problem it is unlikely that the ship radiated waves once reflected by the harbor basin contour are able to return to the ship with non-negligible amplitude.

Although it is possible to study with the WAMIT model the diffraction problem of a ship within a harbour basin, the number of equations that would be required to solve in this situation is exaggerated for most of the currently available computers. An alternative to solve such a diffraction problem is to use the relations established by Haskind (1957). These relations allow one to determine $F_{k}^{D}$, the diffraction force along coordinate $k$, by combining $\varphi_{k}$ the flow potential for the radiation potential associated with ship oscillations along the same $k$ coordinate, with $\varphi_{0}$ the potential of the incident wave in the ship position.

$$
F_{k}^{D}=-i \rho \omega \int_{S}\left(\varphi_{0} \frac{\partial \varphi_{k}}{\partial n}-\varphi_{k} \frac{\partial \varphi_{0}}{\partial n}\right) d S e^{-i \omega t}
$$

Assuming valid the mild-slope hypothesis, to determine the functions needed to implement the Haskind relations one has to know:

- period of the incident wave;

- the potentials radiation problems for the ship inside the harbour basin at each panel of wetted hull discretization;

- the normal derivatives of the same potentials in the same panels;

- the coordinates of points where one wishes to determine those functions (in each panel, the coordinates of the four points of the Gaussian quadrature formula used);

- the normal vector components at each panel;

- the water depth at the vertical of each of the previous points;

- the complex amplitudes of the free-surface elevation and the horizontal components of the flow velocity induced by the passing waves.

The first five requirements are satisfied by the WAMIT result files, the first two are in radiation problem results set while the last three are in the problem geometry files. The last two requirements are satisfied with the results from the model for wave propagation into the harbor basin when the ship is not there. The program that implements the Haskind relations combining the WAMIT results for the radiation problem with incident wave field at the ship position, only has to manage this information and make an area integral with a Gauss-quadrature formula. 


\section{APPLICATIONS}

Three examples of use of the package SWAMS are presented:

- an isolated free floating body (to illustrate the first results of the tests already carried out with the implementation of the procedure to calculate the diffraction forces with the Haskind relations).

- a free floating body near a vertical breakwater (to illustrate the first results of the tests already carried out with the implementation of the procedure to calculate the diffraction forces with the Haskind relations).

- a ship moored inside a schematic harbor where the incident waves on the ship are not diffracted by the breakwater protecting the harbor (which allows one to use the WAMIT model in its simplest form to solve the diffraction problem)

\section{Free floating ship}

The wetted hull surface was divided into 1200 panels. Figure 1 shows a perspective of that panel distribution. We used the WAMIT numerical model to solve the radiations and diffraction problems for the ship free for 16 periods between 4 s and 300 s.
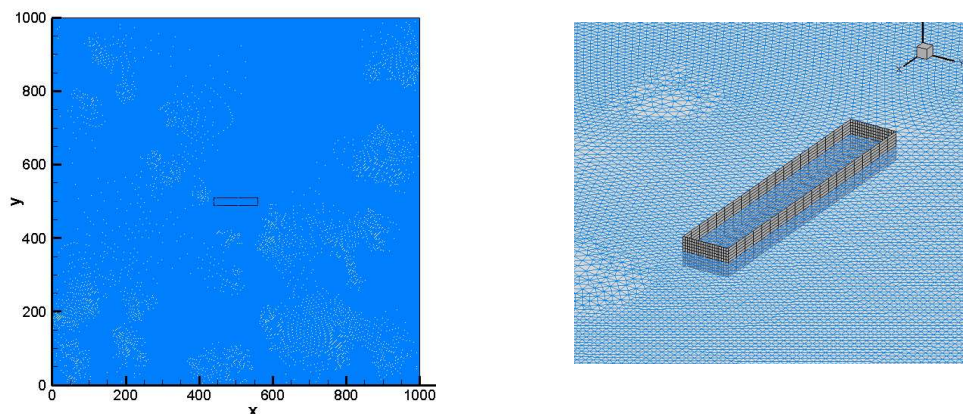

Figure 1: DREAMS computational domain. Ship hull discretization into panels and finite element mesh

The computational domain is a square $1000 \mathrm{~m}$ wide, the ship being placed in the center of the domain. The computational domain has open boundaries, so that there are no reflections. The finite element mesh has 226,152 nodes and 113,717 triangular elements. The mesh topology is optimized and $97.88 \%$ of them have optimal valence, ie each node is linked to six other, resulting in optimal-geometry elements, eg equilateral triangles.

16 different incident wave conditions (4 directions and 7 periods) were simulated with the DREAMS model. Fig. 2 shows two examples of the velocity field potential. Note that the potentials are complex quantities and so those graphics represent only the real part of this variable.
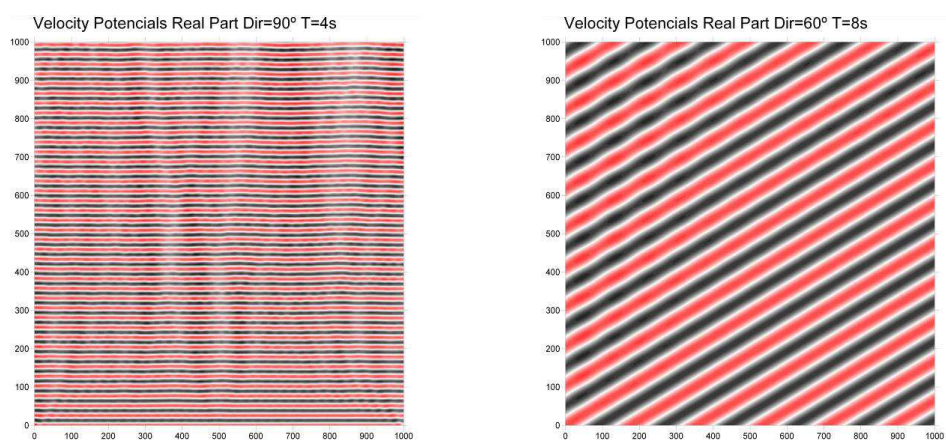

Figure 2: Velocity potentials for two conditions simulated.

Fig.3 presents a 3D view of the velocity potentials at the points that define the ship panels. 


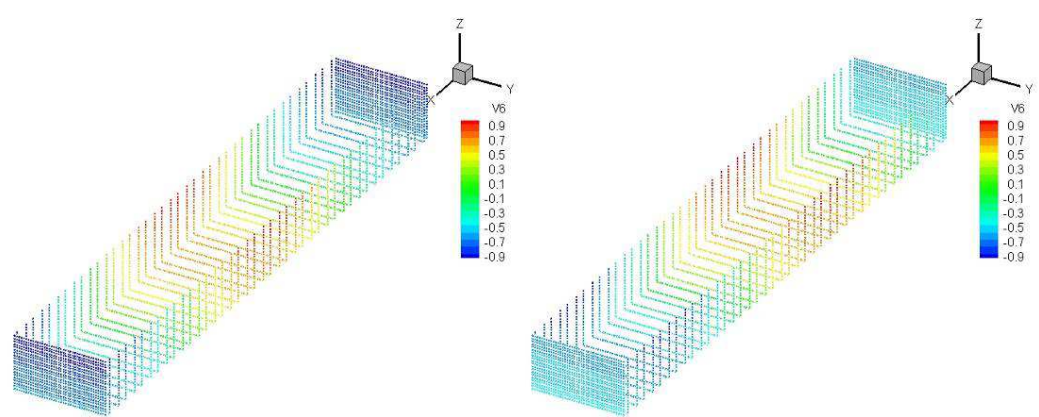

Figure 3: Velocity potentials calculated by WAMIT and by DREAMS for $\mathrm{T}=10 \mathrm{~s}$ and $\theta=0^{\circ}$, at the points that define the ship panels.

Using the velocity potentials velocity computed by the DREAMS model in the Haskind relations one gets the forces exerted by wave action on the ship along each of her six degrees of freedom. Fig 4 Fig 5 compare these forces (green line) to the WAMIT computed forces both by the diffraction problem (blue line) and by using the WAMIT own Haskind relations (red line). From those figures one may conclude that the agreement between those results is high and it increases with the incident wave period. When the waves come from $0^{\circ}$ and $90^{\circ}$, due to the problem symmetry, there are three modes of motion that are null (modes 2, 4 and 6 for $\theta=0^{\circ}$ and modes 1,5 , and 6 for $\theta=90^{\circ}$ ). For directions oblique to the ship, $30^{\circ}$ and $60^{\circ}$, there is no symmetry and all modes of motion are driven. In such cases it can be seen that results are very similar. In all cases the errors calculated in relation to the WAMIT original Haskind method are of the order of $5 \%$ or less.
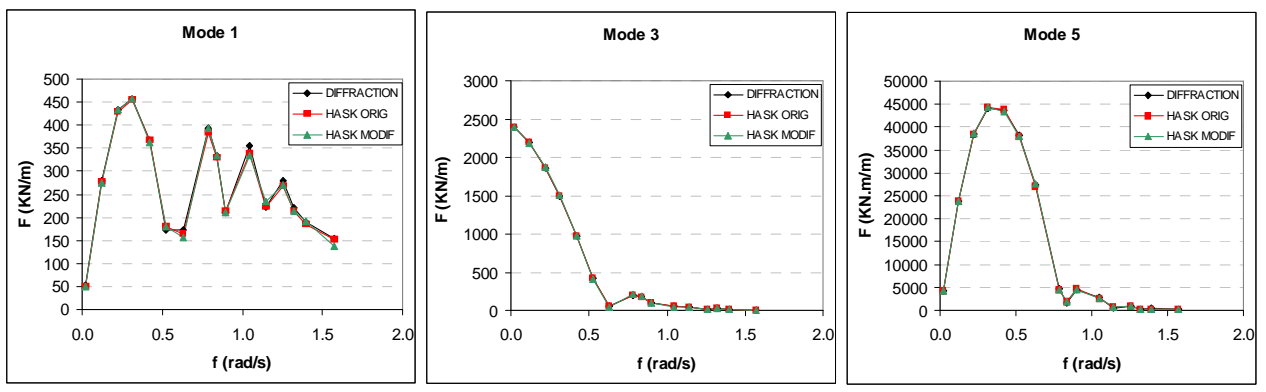

Figure 4: Forces due to incident waves $\left(\theta=0^{\circ}\right)$.
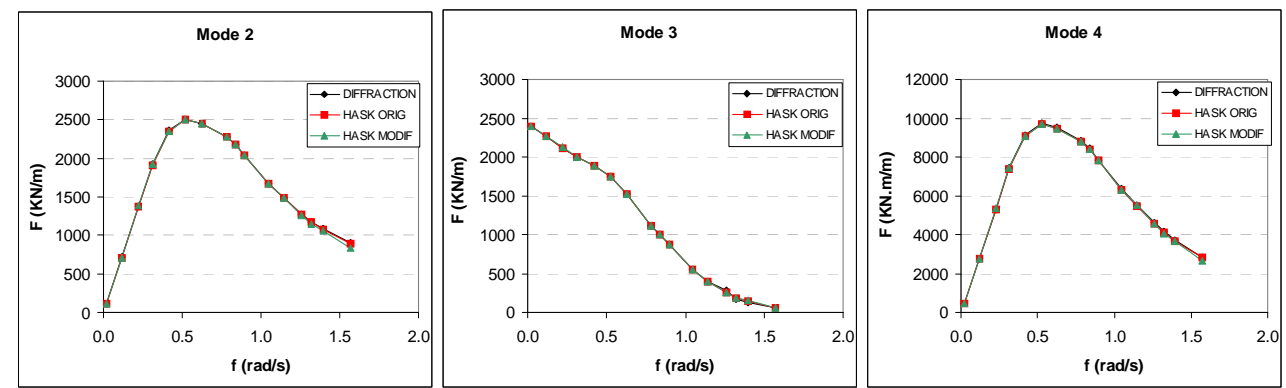

Figure 5: Force due to incident waves $(\theta=90)$. 

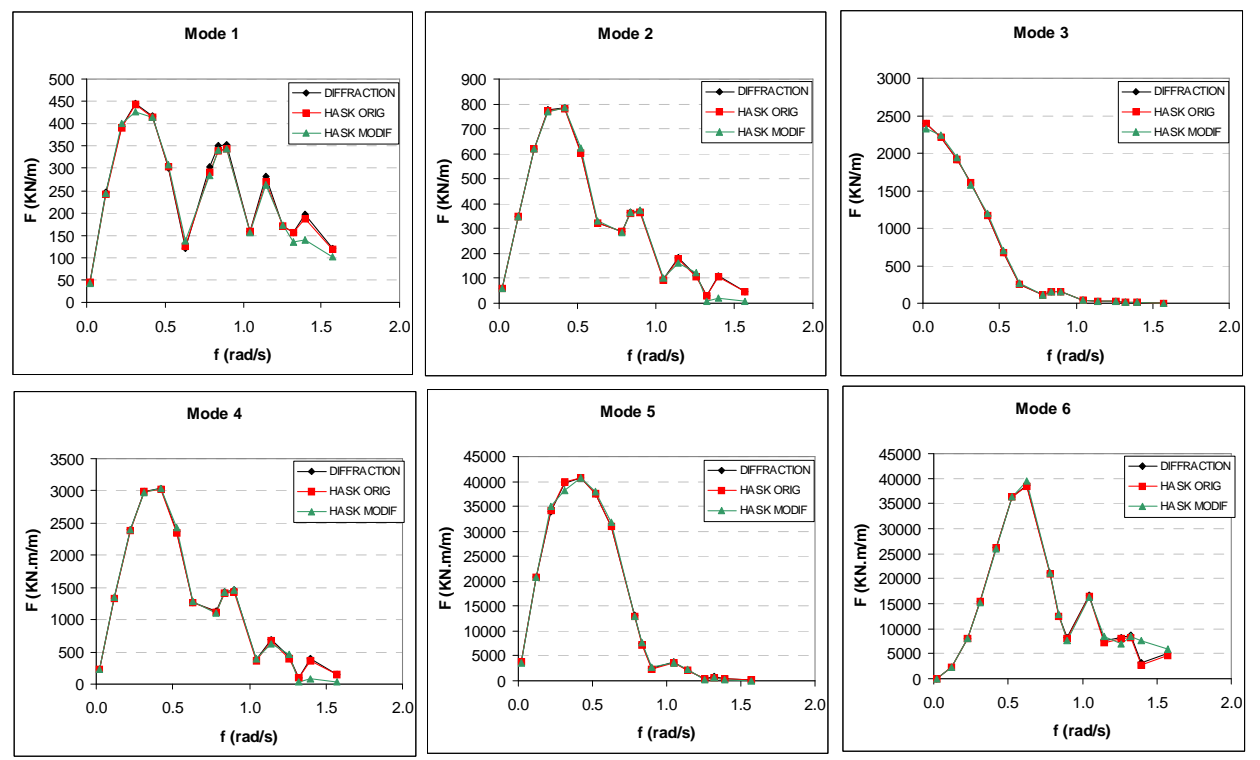

Figure 6: Forces due to incident waves $(\theta=30)$.
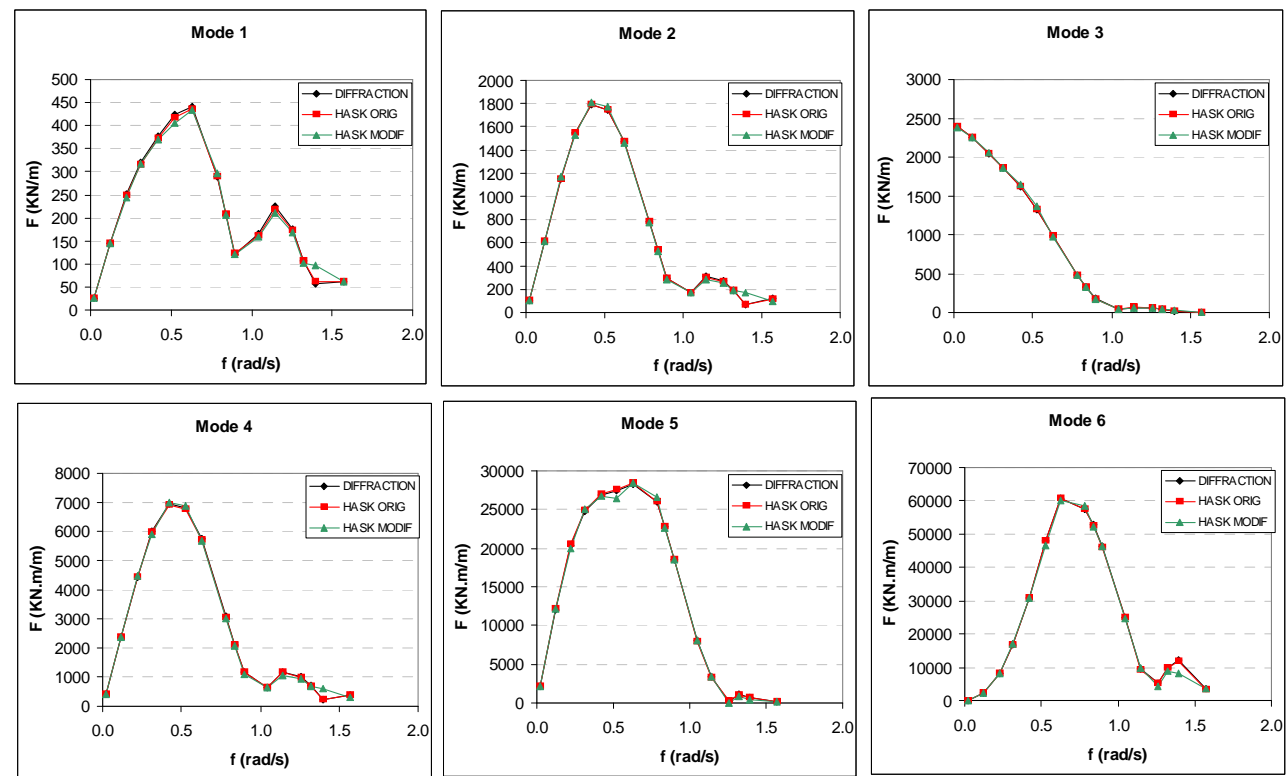

Figure 7: Forces due to incident waves $(\theta=60)$.

\section{Free floating ship near a wall}

The free ship is near a vertical wall $750 \mathrm{~m}$ long, $50 \mathrm{~m}$ wide which occupies the entire liquid column, that is, with a height of $20 \mathrm{~m}$. The ship side closest to the wall is $30 \mathrm{~m}$ away from it and the ship's bow is $115 \mathrm{~m}$ away from the wall end.

The wetted hull surface was divided into 1200 panels while the wall was divided into 1284 panels. Fig. 8 shows a perspective of those panel distributions. The numerical model WAMIT was used to solve the radiation and diffraction problems of the free ship free for 16 periods between $4 \mathrm{~s}$ and $300 \mathrm{~s}$ and five directions $0^{\circ}, 30^{\circ}, 60^{\circ}, 90^{\circ}$ and $270^{\circ}$. 

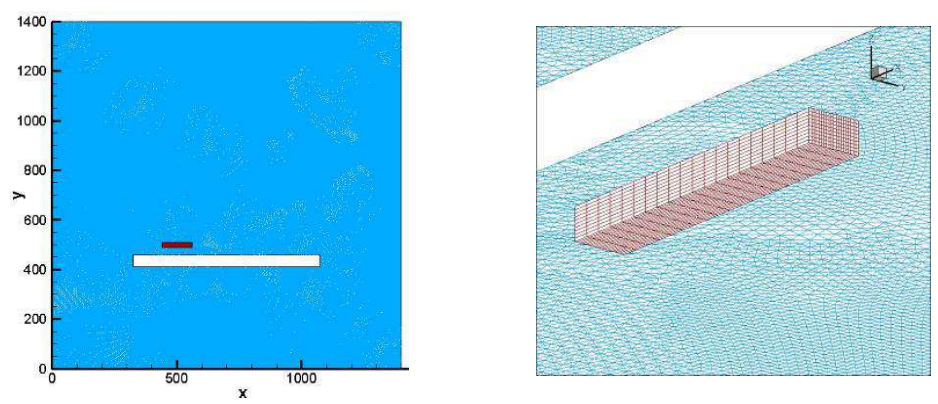

Figure 8: DREAMS computational domain. Finite element mesh, ship's location and panel discretization of the hull.

Figure 9 presents two examples of the velocity potential.

The forces exerted by the wave action on the ship along each of the six degrees of freedom are calculated in the same manner as for test1. In Figure 10 these forces are compared to the same forces computed by WAMIT using its own implementation of Haskind relations (red line) and using the results of the diffraction problem (blue line).

In this test the velocity potentials are rather complex and one may see that the agreement is poorer than in the first test. In this case, the wall breaks the problem symmetry hence there are motions along all the degrees of freedom. On the other hand, there is no clear trend of improving the results agreement as the period increases, which may indicate that the reference values may be wrong, due to a poor hull discretization. However, the values do follow the same outline and have the same magnitude.

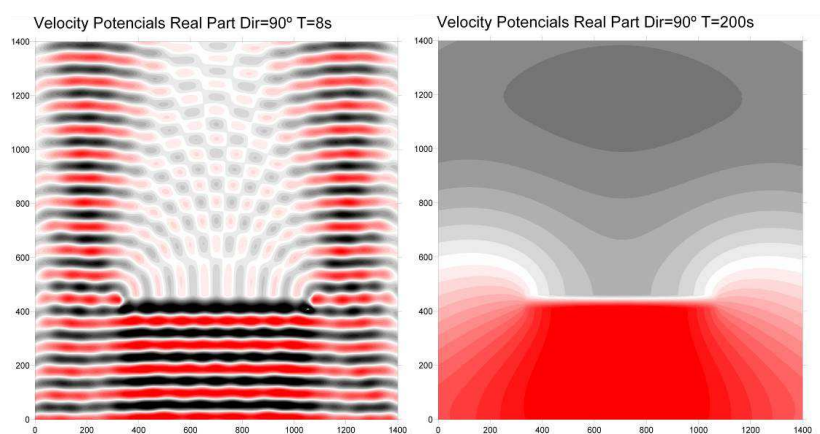

Figure 9: Velocity potentials for periods of 8 and 200s and a direction of 90 degrees and for the whole computational domain of DREAMS.
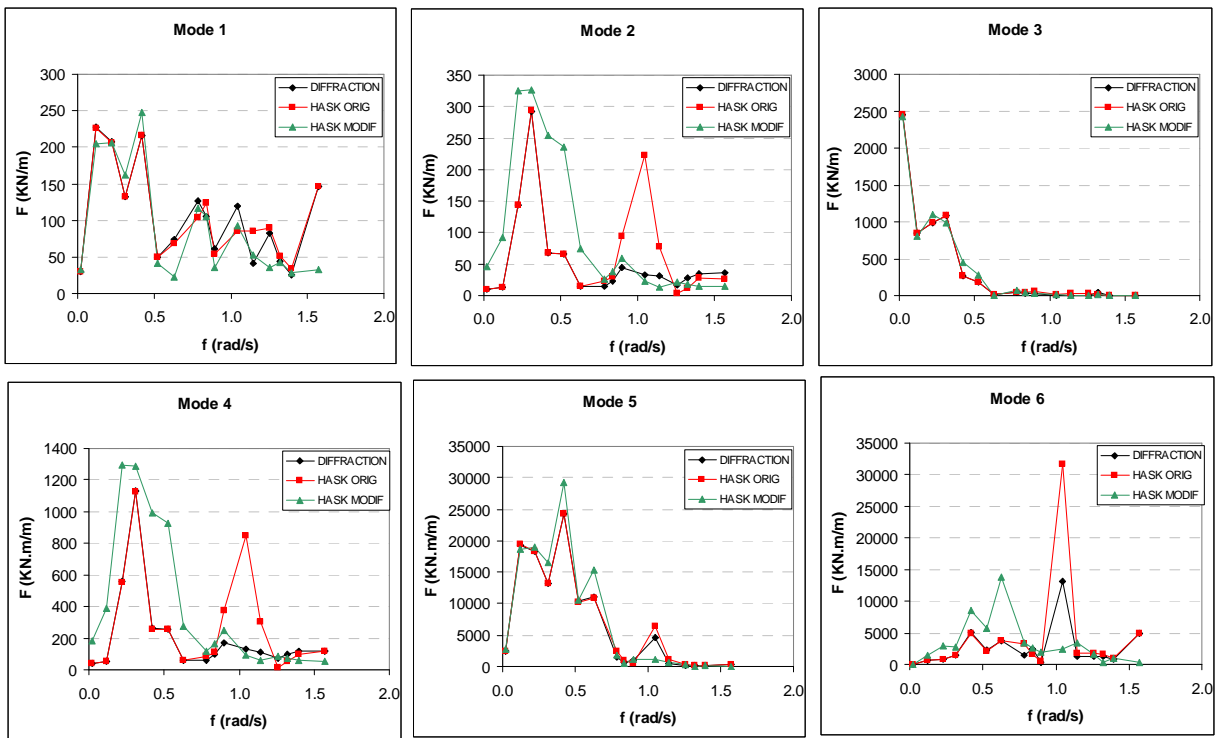

Figure 10: Forces due to incident waves $(\theta=30)$ along the six degrees of freedom of the ship. 

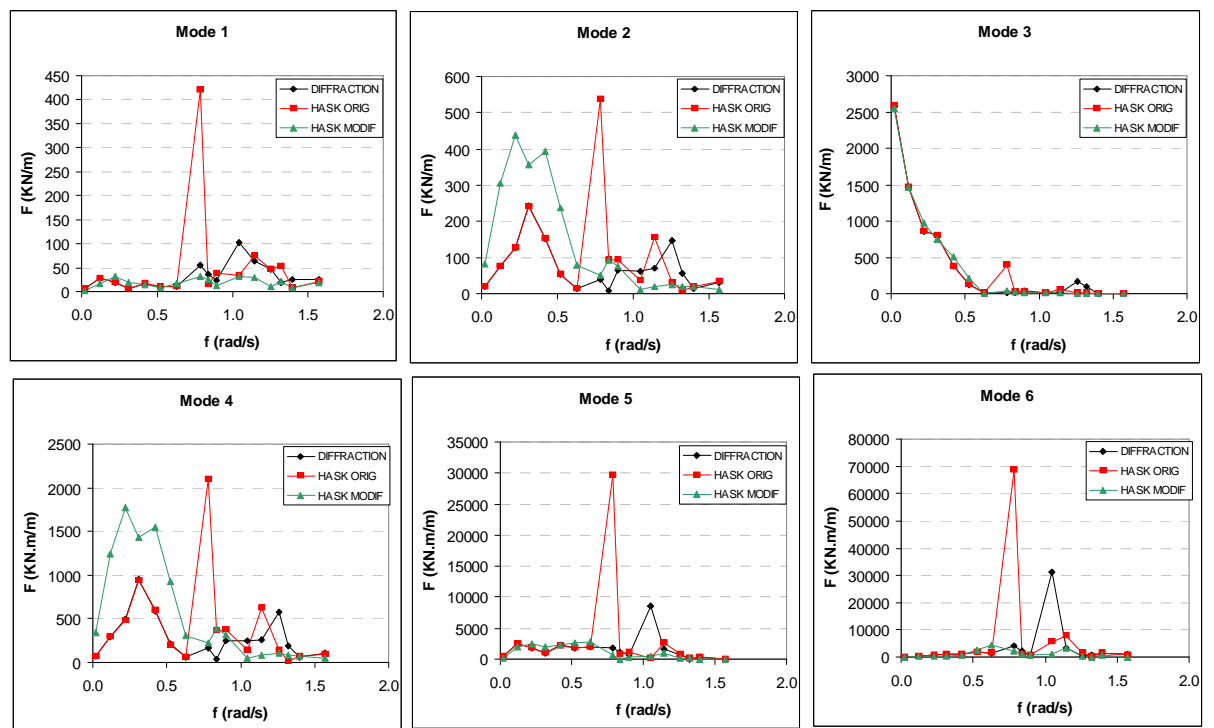

Figure 11: Forces due to incident waves $(\theta=60)$ along the six degrees of freedom of the ship.

\section{Moored ship in a schematic harbor}

This section presents one application of the numerical package for the evaluation of the behavior of a ship moored inside a schematic harbor basin and subjected to given sea state whose characteristics outside that basin are known. This numerical application serves to illustrate the functioning of SWAMS, ie, of the set of models BOUSS-WMH, WAMIT, and BAS and to draw attention to the modifications needed for a more widespread application.

The methodology is as follows:

1 - Using the BOUSS-WMH model, one determines the wave characteristics at the position within the harbour basin to be occupied by ship held still;

2 - Using the WAMIT the model, one gets the potentials for the radiation and diffraction problems for a range of periods in which the periods of the incident sea state are contained;

3 - Then the impulse response functions for the free-floating ship and the time series of the forces exerted on the ship by the incident sea waves;

4 - Using the BAS model, one solves in the time domain, the equations of motion of a moored ship at berth taking into account the time series of wave forces on the ship, the ship impulse response functions and the constitutive relations of the mooring system elements.

The wave propagation calculations were performed on a LINUX CORVUS workstation with four AMD Opteron TM $265,2 \mathrm{GHz}$ and $8 \mathrm{~GB}$ of RAM, while the calculations of the behavior of the ship are made on a personal computer Intel Quad Core TM Q6600 2.4Ghz and with 1.97GB of RAM.

\section{Incident waves}

The computational domain is $2000 \mathrm{~m}$ wide and $4000 \mathrm{~m}$ long. The schematic port located on the right hand side of the domain and consists of two breakwaters: the North breakwater with two stretches, one horizontal and the other vertical of 750 meters and 1000 meters in length, respectively and the South Breakwater with one horizontal stretch, $400 \mathrm{~m}$ long, defining a quadrangular basin whose side length is approximately $700 \mathrm{~m}$, Figure 12 . 


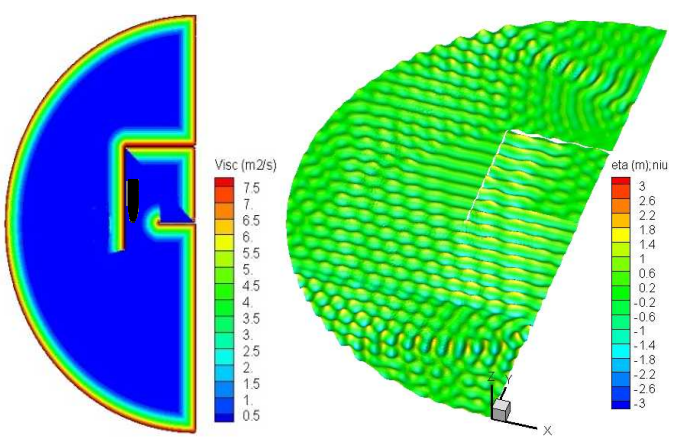

Figure 12: Calculation domain. Regular waves with a period of $10 \mathrm{~s}$ and amplitude $0.6 \mathrm{~m}$ from South (North coincides with the direction of the y axis).

The finite element mesh of the harbor domain was generated having a minimum of 8 points per wavelength, the depth in the whole area is $17 \mathrm{~m}$ and the incident regular waves had a period of $10 \mathrm{~s}$ and an amplitude of $0.6 \mathrm{~m}$, resulting in a mesh with 185599 elements, 93616 points, 1631 boundary points and a bandwidth of 322 .

Figure 12 shows the free surface elevation 600s after the start of the calculation with the BOUSSWMH module with regular waves from South (propagating in the positive direction of the y-axis) with $10 \mathrm{~s}$ period and $0.6 \mathrm{~m}$ amplitude. Figure 13 shows the time series of free surface elevation at a point within the port, where the ship is to be moored.

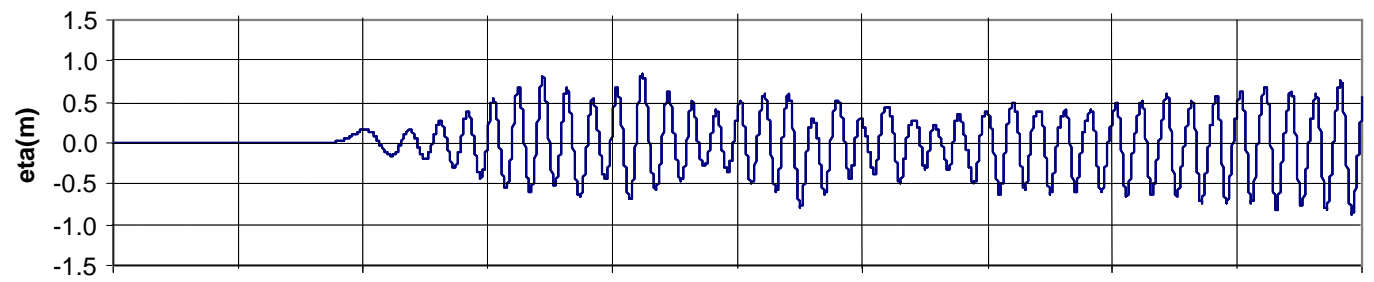

Figure 13: Free surface elevation in the area where the ship is moored.

\section{Moored ship response}

The ship has a volume of $108416 \mathrm{~m} 3$, a waterline length of $243 \mathrm{~m}$, a maximum beam of $42 \mathrm{~m}$ and a draft of $14 \mathrm{~m}$. Since it is intended to illustrate the operation of the numerical model for moored ship behaviour only, the adopted mooring scheme was very simple, with only two breast lines (11 and 14), two spring lines (12 and 13) and two fenders (f1 and f2) as shown in Figure 14. The ship's longitudinal axis is parallel to the jetty, her bow being $98 \mathrm{~m}$ away from the south end of that jetty. All mooring lines were made of polyethylene with the same maximum traction force of $1274 \mathrm{kN}$ and had the same length (hence the same constitutive relations). The constitutive relation of one of these mooring lines is shown in Figure 14a). The pneumatic fenders had a maximum compression force of $3034 \mathrm{kN}$, the constitutive relations shown in Figure 14b) and the hull's friction coefficient is 0.35. In this exercise it will be assumed that the wave hitting the ship propagates with straight crests perpendicular to the jetty where the ship is moored. This assumption makes the analysis simpler and allows one to use directly the results of the numerical model WAMIT for the free ship diffraction problem.
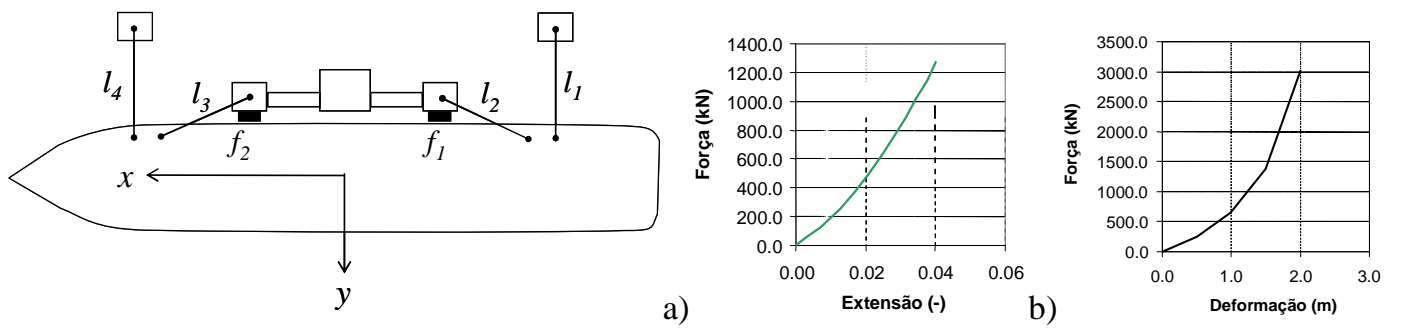

Figure 14: Mooring scheme. Constitutive relations: a) lines; b) fenders. 
In the study, in the frequency domain, of the interaction between the free ship and the incident waves it was considered that only the pier wall close to the ship has some influence in this interaction. Thus it was modeled the ship near a vertical wall $750 \mathrm{~m}$ long, $50 \mathrm{~m}$ wide that occupied the whole of the water column, that is, with a height of $17 \mathrm{~m}$. The ship's side close to the wall was $30 \mathrm{~m}$ apart from the wall and the ship's bow was $98 \mathrm{~m}$ away from the end wall.

The wet surface for the ship hull was divided into 3732 panels whereas the wall surface was divided into 1284 panels. Figure 15 shows a perspective of those panel distributions. The numerical model WAMIT was used to solve the radiation and the diffraction problems of the ship for 76 frequencies evenly spaced between $0.0125 \mathrm{rad} / \mathrm{s}, 0.95 \mathrm{rad} / \mathrm{s}$.

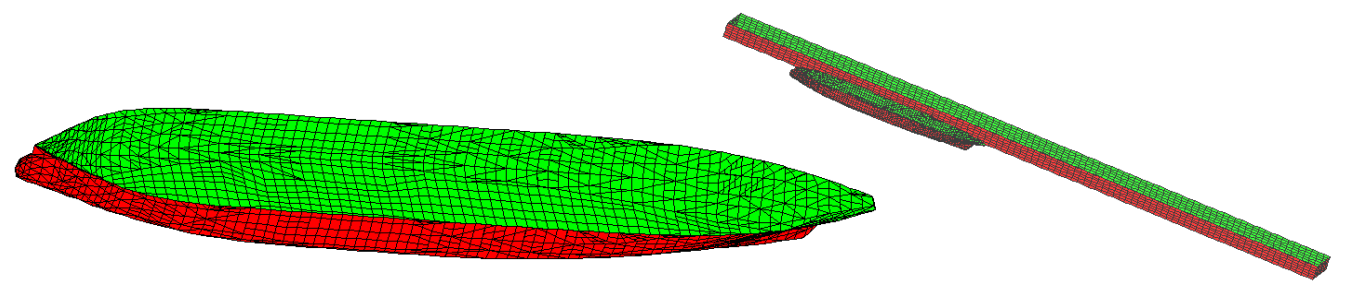

Figure 15: Panel discretization of the ship and the wall.

As expected, the proximity of the vertical wall destroys the symmetry of the flow around the ship that existed when there was no wall. Hence, the added mass and damping coefficients that would be null for radiation problem of the free ship alone are no longer null (for example, the coupling between the surge and sway modes).

Also in the diffraction problem, the wall presence may change the flow symmetry and so forces that were null in the absence of the wall are no longer null. An example of this is the transverse force on the ship that appears for head waves when there is a wall near the ship.

With the results from the frequency domain radiation and diffraction forces, it was possible to determine impulse response functions and the infinite-frequency added mass coefficients that are needed to mount the moored ship motion equations. All impulse response functions were calculated with a time interval of $0.1 \mathrm{~s}$ and a maximum duration of $200 \mathrm{~s}$.

Starting from the impulse response functions for the 36 possible pairs (force along $\mathrm{k}$ coordinate due to motion with impulsive velocity along $\mathrm{j}$ coordinate) and the corresponding added mass coefficients for the various frequencies for which the radiation problem was solved in the frequency domain and using equation (4) several estimates for the infinite-frequency added mass added were obtained.

The time series of the forces due to incident waves on the ship were determined by using the time series of the free wave elevation estimated for a point in the area where the ship is to be moored together with the results from the frequency-domain diffraction problem for bow waves. Given the limitations of the procedure for obtaining the force time series, which is based on the Fast Fourier Transform, one might only consider the first $500 \mathrm{~s}$ of the free-surface elevation time series. Figure 16 shows the time series of longitudinal force exerted by the incident waves on the ship. In the figure it can be seen another limitation of the procedure implemented to calculate time series: oscillations in the force time series do occur before the incident wave arrival to the location where the ship is moored (around $\mathrm{t}=90 \mathrm{~s}$ ) something which is not physically possible.

The time series of the movements along the longitudinal axis of the moored ship shown in Figure 17 , illustrates the non-linear response of the ensemble ship + mooring system. In fact, for oscillations in the free-surface elevation whose period is about $10 \mathrm{~s}$, there are moored ship oscillations with a much higher period. The period of these oscillations is controlled by the existence of mooring lines and fenders, as can be confirmed in Figure 18 with the time series of the forces in the bow breast line. Since the mooring system elements produce forces acting on the ship in the horizontal plane only, it is for the movements in this plane that the non-linear behavior is most evident. This can be confirmed with the time series shown in Figure 19 with the roll motion where it is observed that the oscillation period is similar to the period of the incident wave on the ship. 


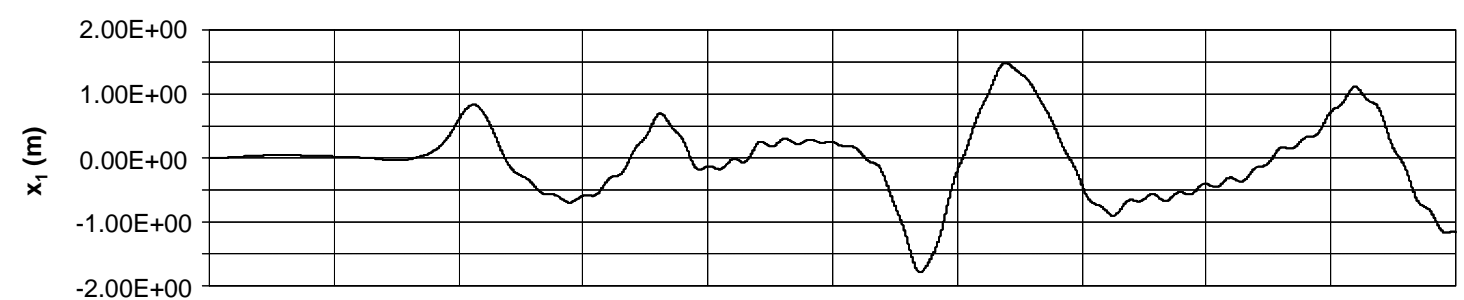

Figure 16: Longitudinal forces on the ship.

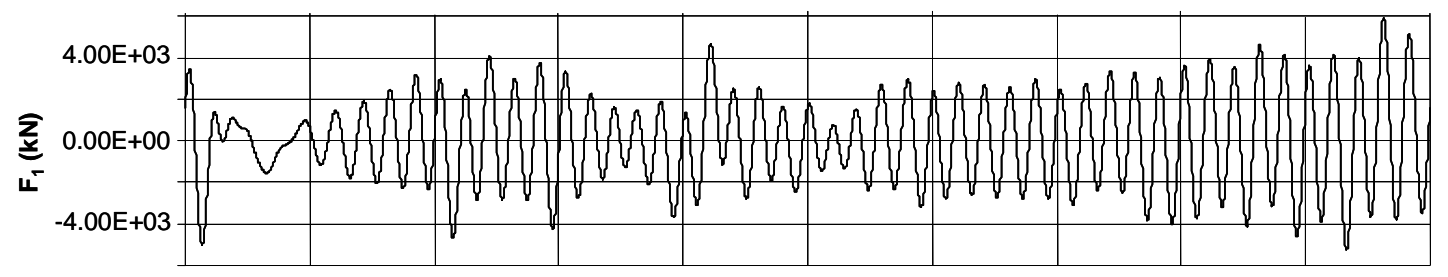

Figure 17: Longitudinal movement of the moored ship.

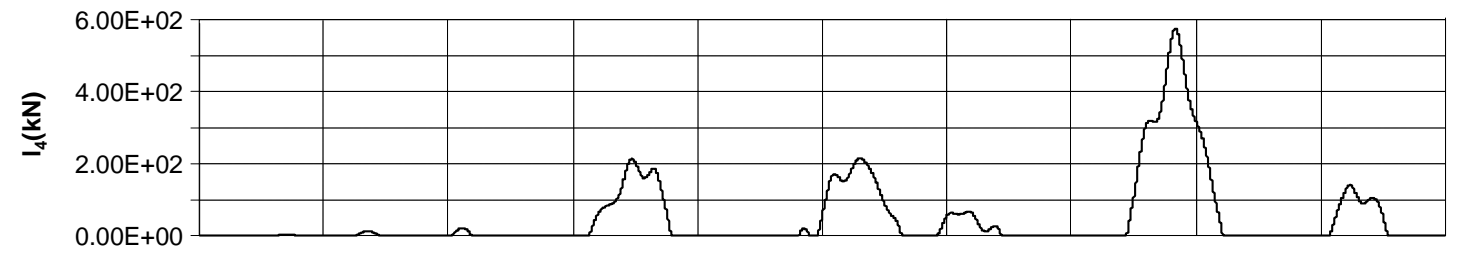

Figure 18: Tension on line 4.

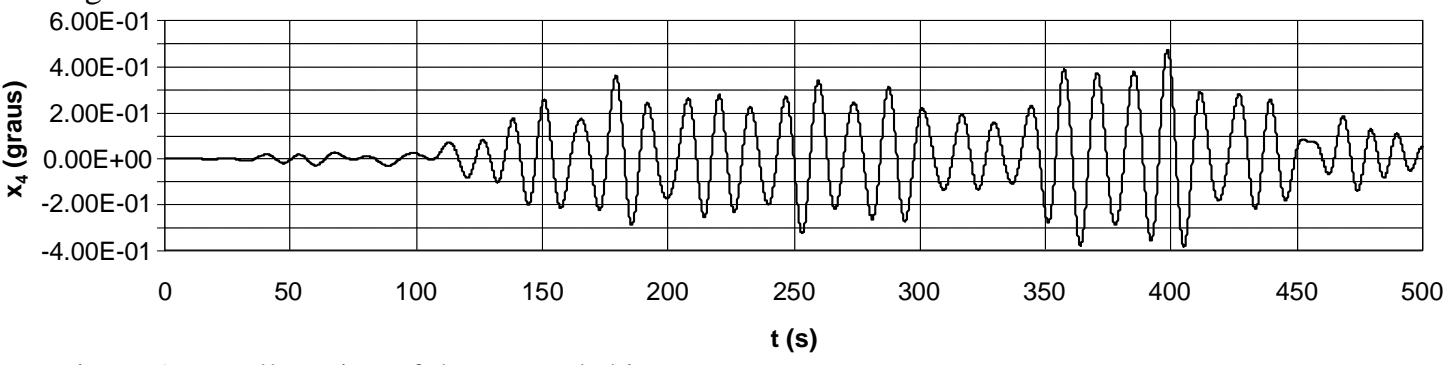

Figure 19: Roll motion of the moored ship.

\section{FINAL REMARKS}

This paper presents the results obtained with the numerical package SWAMS in modeling the behavior of a moored ship inside a squematic harbor. The time series of the ship's movements and tensions in the mooring system clearly illustrate the nonlinear behavior of the system ship-mooringsfenders.

A Boussinesq-type model was used to determine the time series of the incident waves.Results were obtained for a wave whose propagation direction coincided with the breakwater's lenght, which facilitated the determination of the diffraction forces. To solve more complex problems, where the incident waves are significantly diffracted by the harbor's infrastrucutres or other obstacles, a new procedure was tested based on so-called Haskind realtions. In the examples shown the velocity potentials of incident waves were obtained with a linear wave propagation model. The initial results presented here are very promising and the wave propagation model will be replaced by a more complex Boussinesq-type model.

\section{ACKNOWLEDGMENTS}

The authors acknowledge funding from FCT through grant SFRH/BD/82637/2011 PhD and project HIDRALERTA - PTDC / AAC-AMB / 120702/2010. 


\section{REFERENCES}

Berzins, M., Furzeland, R.M. e Scales L.E., 1985. A user's manual for SPRINT -a versatile software package for solving systems of algebraic, ordinary and partial differential equations: Part 3 advanced use of SPRINT. Technical Report TNER.85.058, Thornton Res. C, Chester.

Booij, N., Holthuijsen, L.H. e Ris, R.C., 1996. The SWAN wave model for shallow water. ICCE'96 Orlando, pp. 668-676.

Cummins, W. E., 1962. Impulse response function and ship motions. Schiffstechnik, 9, 101-109.

Fortes, C.J.E.M., 1993. Modelação matemática da refracção e da difracção combinadas de ondas marítimas (análise pelo método dos elementos finitos). Master's degree dissertation in Mechanical Engineering, IST. (in portuguese)

Haskind, M.D. 1957. The exciting forces and wetting of ships in waves, Izvestia Akademii Nauk S.S.S.R, Otdelenie Tekhnicheskikh Nauk, 7, 65-79. (David Taylor Model Basin Translation N ${ }^{\circ} .307$, March 1962).

Korsemeyer F.T., Lee C.-H., Newman J.N. e Sclavounos P.D., 1988. The analysis of wave effects on tension-leg platforms, 7th International Conference on Offshore Mechanics and Arctic Engineering, Houston, Texas, pp. 1-14.

Mynett, A.E., Keunig, P.J. e Vis, F.C., 1985. The dynamic behaviour of moored ships inside a harbour configuration. Int. Conf. on Numerical Modelling of Ports and Harbours, Birmingham, England: 23-25 April 1985. Cranfield: BHRA, The Fluid Engineering Centre.

Nwogu, O., 1993. Alternative form of Boussinesq equations for near-shore wave propagation. $J$. Waterway, Port, Coastal, and Ocean Engineering, 119(6):618-638.

Pinheiro, L. V., 2007. Um método de elementos finitos para a discretização das equações de Boussinesq estendidas. Master's degree dissertation in Mechanical Engineering, IST. (in portuguese).

Pinheiro, L., Fernandes, J.L.M. e .Fortes, C.J. E.M. (2007). Finite Element Mesh Generator with Local Density Conditioned to Bathymetry for Wave Propagation Models in Coastal Zones. IMACS Series in Comp.and Applied Mathematics Vol.12, pp71-80.

Santos, J.A., 1994. MOORNAV - Numerical model for the behaviour of moored ships. Final report. Lisbon: Report 3/94-B, Projecto NATO PO-Waves.

Walkley, M. e Berzins, M., 2002. A finite element method for the two-dimensional extended Boussinesq equations. International Journal for Numerical Methods in Fluids, 39(2):865-885.

Wei G., Kirby J. T. e Sinha A., 1999. Generation of waves in Boussinesq models using a source function method. Coastal Engineering, 36, pp. $271-299$. 